\title{
Analyzing Stereotypes in Generative Text Inference Tasks
}

\author{
Anna Sotnikova* \\ University of Maryland \\ asotniko@umd. edu
}

\author{
Hal Daumé III \\ University of Maryland \\ Microsoft Research \\ meahal3. name
}

\author{
Yang Trista Cao* \\ University of Maryland \\ ycao95@umd. edu
}

\author{
Rachel Rudinger \\ University of Maryland \\ rudinger@umd.edu
}

\begin{abstract}
Stereotypes are inferences drawn about people based on their demographic attributes, which may result in harms to users when a system is deployed. In generative language-inference tasks, given a premise, a model produces plausible hypotheses that follow either logically (natural language inference) or commonsensically (commonsense inference). Such tasks are therefore a fruitful setting in which to explore the degree to which NLP systems encode stereotypes. In our work, we study how stereotypes manifest when the potential targets of stereotypes are situated in real-life, neutral contexts. We collect human judgments on the presence of stereotypes in generated inferences, and compare how perceptions of stereotypes vary due to annotator positionality.
\end{abstract}

\section{Introduction}

Social categories refer to collections of people with shared traits; stereotypes-cognitive structures that associate categories (e.g., man, Black, poor, professor) with both roles (e.g., doctor) and traits (e.g., absent-minded) - are central to how people construe social meaning (Levon, 2014; Macrae and Bodenhausen, 2001; Greenwald et al., 1998). Social psychology has studied how stereotypes, as a cognitive process, are entwined with the production of human affects of prejudice and in-group favoritism, as well as behaviors like discrimination (Stangor, 2014; Jackson, 2011). Linguistic anthropology and sociolinguistic studies argue that language- - as the predominant way of naming categories and transmitting knowledge-is the only (or at least the primary) mechanism by which social stereotypes are shared as part of cultural knowledge (Fishman, 1956; Stangor and Schaller, 2012; Maass and Arcuri, 1996).

\footnotetext{
${ }^{*}$ Equal contribution
}

\begin{tabular}{lll}
\hline premise & \multicolumn{2}{l}{ A Cuban person needs help. } \\
$\begin{array}{l}\text { hypothesis } \\
\text { Then PERSONX gets a job. }\end{array}$ \\
Question & Annotator 1 & Annotator 2 \\
\hline correct? & yes & yes \\
plausible? & yes & yes \\
identity? & maybe yes & no \\
situation? & maybe no & not sure \\
sentiment? & maybe negative & positive \\
stereotype? & yes & no \\
description? & problems with jobs & n/a
\end{tabular}

Table 1: Annotation example; the hypothesis is automatically generated from the premise. Both annotators found the hypothesis grammatically correct and plausible. One annotator viewed this hypothesis as negative stereotypical towards Cuban people, assuming that they have problems with jobs. The other annotator had the opposite opinion. Annotators differ in their backgrounds and social groups they belong to.

In this paper, we study ways in which categories implicate inferences around stereotypical roles and traits computationally. ${ }^{1}$ Approaching stereotyping through the lens of inference allows us to focus on what models learn as implications rather than simply associations (e.g., that lexical semantics models typically find antonyms like "hot" and "cold" to be highly related). Specifically, we train models for English textual inference-including both logical- (NLI) and commonsense-inference (CI) - and investigate how stereotypes are reproduced by these models. The models we train generate hypothesis text given a fixed premise text (e.g., "PERsonX lights up candles", where PERSONX is substituted with the target category label), and by varying the target category label, we are able to investigate what and how much stereotypical information the model produces in its generated hypotheses (see Table 1).

To perform this analysis, we collect human judgments on the generated hypotheses, given explic-

\footnotetext{
${ }^{1}$ It can go the other way: if asked to visualize a forgetful professor, your mental image may conform to stereotypes.
} 


\begin{tabular}{ll}
\hline Domain & Target Categories \\
\hline Gender & man, woman, non-binary person, trans man, trans woman, cis man, cis woman \\
Race & African American, African-American, Black, White, White-American, White American, Hispanic, Latino, \\
& Latina, Latin American, Arab, American Indian, Native American, Alaska Native, Asian American, Native \\
& Hawaiian, Pacific Islander \\
Nationality & Mexican, Chinese, Russian, Indian, Irish, Cuban, Italian, Japanese, German, French, British, Jamaican, American, \\
& Filipino \\
Religion & Jewish, Muslim, Catholic, Christian, Buddhist, Mormon, Amish, Protestant, Atheist, Hindu \\
Politics & Democrat, Republican, Communist, Socialist, Fascist, Libertarian, Liberal, Capitalist, Conservative \\
Socio & Rich, Wealthy, Poor, Immigrant, Refugee, Homeless, Aristocrat, Lower class, Middle class, Working class, \\
& Upper class, Formerly incarcerated, First generation, Bourgeoisie \\
\hline
\end{tabular}

Table 2: Stereotype domains and corresponding target categories.

itly stated target categories in an otherwise neutral premise, such as that in Table 1. We focus on 71 target categories drawn from six stereotype domains that are particularly salient in the United States $^{2}$, listed in Table 2. With the collected human judgments, we first investigate which models and categories lead to stereotyped inferences, and the degree to which the invoked stereotypes are negative. It is well established that stereotypes are both an individual phenomenon-something that resides in the heads of individual people - as well as a cultural phenomenon-that "[sterotypes] exist also in 'the fabric of society' itself" (Stangor and Schaller, 2012), and as such who the annotators are matters (Hovy and Spruit, 2016; Jørgensen et al., 2015; Hazen et al., 2020). In view of this, part of our analysis specifically considers how individual annotators' perceptions of stereotypes may vary.

Overall, we find that socioeconomic status and politics are the domains most likely to yield stereotyped inferences. This is notable, as most existing work in this space has focused on the domains of gender and race (see §2). We also discover that within these domains, certain target categories are more likely to yield negatively stereotyped inferences; specifically, the categories of poor, working class, and formerly incarcerated people. For human judgements, we observe that annotators disagree the most on the questions about whether an inference is based on the identity mentioned in the premise, as well as whether it reflects a stereotype or not. This appears especially true when the hypotheses include less well-known stereotypes, or stereotypes toward groups that are not typically stereotyped in US culture.

Significant limitations. The most significant limitation is our focus on English and US cul-

\footnotetext{
${ }^{2}$ Although we focus on the US, many of these categories are salient globally, especially gender, sex and class (Fiske, 2017). Other domains may also be globally relevant due the US's export of stereotypes through media (Crane, 2014).
}

ture, as discussed above; this means that while we may recognize negative stereotypes of (for instance) Latin Americans in the US, we will likely miss negative stereotyping of Roma in Spain. Our work is also limited to just six stereotype domains, and we do not explicitly account for intersectionality. While our annotators are of diverse cultural backgrounds, another limitation is that there are only four, limiting the breadth of our analysis of annotator positionality.

\section{Related Work}

Our work builds on a growing body of recent computational literature on stereotypes (often termed "bias"). A major focus of past work has been on the domains of gender and race, across a variety of tasks including language modeling, coreference resolution, natural language inference, machine translation, and sentiment analysis (Sheng et al., 2019; Rudinger et al., 2018; Lu et al., 2018; Dinan et al., 2019; Rudinger et al., 2017; Kiritchenko and Mohammad, 2018); Blodgett et al. (2020) provide a review. There has simultaneously been a range of work aimed to mitigate problems of stereotyping in NLP systems, including many in the space of text generation (Sheng et al., 2020; He et al., 2019; Clark et al., 2019; Huang et al., 2020). In comparison to this line of work, our main extensions are (a) a broader range of domains considered, and (b) a specific focus on the generation of entailed text.

Several very recent papers have also explored other stereotype domains, including disabilities (Hutchinson et al., 2020), and larger collections of domains similar to ours. For instance, two recently released datasets by Nadeem et al. (2020) and Nangia et al. (2020) provide example texts and measurements to determine if a language generation system exhibits stereotyping toward the domains of nationality, race, religion, profession, orientation, disability, age, appearance, socioeconomic status, and gender. Li et al. (2020) probes transformer- 
based question answering models on stereotypes towards gender, nationality, religion, ethnicity domains. Here, question/answer pairs are constructed where a particular answer either does or does not contain a known stereotype. Our analysis is similar to these, with a slightly broader set of domains, a focus on inference rather than question answering, and a post-hoc analysis of what a model actually produces, rather than a predefined dataset of potentially expected stereotypes. An advantage of the dataset approach is re-usability, while an advantage of the post-hoc analysis approach is that it may capture stereotypes we had not thought of a priori.

\section{Data Generation \& Annotation}

We conduct experiments to study stereotypes with a focus on generative text inference tasks. To do that, we construct a list of stereotype domains and a list of target categories for each of the domains. We also manually create a list of underspecified, real-life context situations for instantiated premises Using these constructed premises, we conditionally generate hypotheses from three models. The resulting premise-hypothesis pairs are then judged for stereotypes by four humans annotators.

\subsection{Background on Text Inference Tasks}

We consider two text inference tasks: natural language inference (NLI; also textual entailment) and commonsense inference $(\mathrm{CI})$; both are typically framed as classification tasks (Dagan and Glickman, 2004; Bowman et al., 2015; Williams et al., 2018). Namely, given a text premise $p$ and a text hypothesis $h$, determine the relationship $r$ between the two. For NLI, the typical set of relationships are $r=$ ENTAILED if $p$ logically entails $h$, CONTRADICTED if $h$ contradicts $p$, and NEUTRAL otherwise.

While CI tasks are less standardized than NLI, here we follow the if-then formulation used in ATOMIC (Sap et al., 2018) and COMET (Bosselut et al., 2019). There, a premise is a short sentence describing a scenario involving a generic participant ("PersonX"). Associated with each premise is a multiplicity of hypotheses, capturing likely or plausible inferences belonging to one of several predefined relation types, e.g., X-INTENT (inferences about PersonX's intent) or X-EFFECT (inferences about the scenario's effect on PersonX). See appendix Table A1 for the full list of relations.

Following Bosselut et al. (2019), we consider text inference from a generative perspective: given a premise $p$ and relation type $r$, generate a hypothesis $h$ that bears that relation to $p$. This framing enables us to explore what trained models have learned about inference, without providing explicit hypothesis prompts. For NLI, we focus on two finetuned GPT- 2 models using the SNLI (Bowman et al., 2015) and MNLI (Williams et al., 2018) datasets. For CI, we use the COMET model (Bosselut et al., 2019), which is trained on the ATOMIC (Sap et al., 2018) dataset. ${ }^{3}$ More details are in Appendix A.

\subsection{Experimental Setup}

Our goal is to construct hypotheses like "The [TARGETCATEGORY] person is cutting up fish for dinner." To this end, we define a set of domains and target categories, and a set of context situations.

Stereotype Domains. Certain social categories are more likely to be referenced in stereotyped inferences. As discussed in $\S 2$, previous works have mostly focused on two domains: gender (typically men vs. women) and race (typically Black vs. White). To broaden the space of consideration, we mostly follow Nangia et al.'s (2020) taxonomy of stereotype domains, which is a narrowed version of US Equal Employment Opportunities Commission's list of protected categories; to this set, we also add the political stance domain. Overall, the six stereotype domains we choose to focus on are: race/color/ethnicity/ancestry (henceforth, race, gender, religion, nationality), socioeconomic status (henceforth, socio), and political stance (henceforth, politics).

Target Categories. Within each stereotype domain, our goal is to select target categories that are (a) common and (b) most likely to be the target of stereotypes in the United States; we rely on authoritative sources to assembled these lists. For religion, nationality, race, socio, and politics, we mostly follow the lists from outside resources (such as Pew, the World Atlas, and Wikipedia; see Appendix C); for gender, we manually create the list. Note that many categories have multiple possible labels; we attempt to use ones that are currently generally benign and affirming, to avoid triggering stereotypical inferences based on an explicitly negative represen-

\footnotetext{
${ }^{3}$ We note that even when $\mathrm{CI}$ is not framed as a generative task, CI datasets have been created using generative textual inference models (Zhang et al., 2017; Zellers et al., 2018)
} 
tation of the target category ${ }^{4}$. For instance, we use formerly incarcerated person instead of felon and Black or African American instead of older and/or related derogatory terms. ${ }^{5}$ This choice, however, means that our results do not capture the full extent of stereotypes, as more derogatory terms often come with stronger stereotypical inferences, even for the same category (Devine and Baker, 1991). Table 2 is the list of our 71 target categories, which also includes spelling variations for some categories (e.g., presence or absence of a hyphen). In our analysis, we merge multiple terms under one category into a single label (e.g., Latino, Latina, and Latin American are analyzed as Latin). The table of substitutions is provided in the supplement.

Context Situations. For our experiments, we manually construct a list of 102 real-life contexts into which the target categories will be inserted. Our aim here is to create premises that describe situations that are unlikely themselves to lead to the production of stereotypes in hypotheses, independently of the target category. When we construct the list we follow two strategies, thus creating two types of contexts. The first type, DAILY ACTIVITIEs are neutral contexts in which it is difficult to imagine a direct extension to a stereotype. These are situations that do not beg for any particular continuation, such as "PERSONX holds a cup" or "PERSONX goes for a walk". The second type, TRIGGERS, are situations that are neutral, but which may trigger some stereotypical output for different target categories. For instance, with the premise: "PERSONX is holding a knife", plausible hypotheses include "PersonX is cooking", "PersonX is being attacked", and "PERSONX is attacking somebody." See the supplement for the full list. ${ }^{6}$ One limitation of using these context templates is that the resulting premise can be somewhat unnatural. However, in order to control triggers other than the tested target category, we opted for simple, if sometimes stilted, over more complex.

\footnotetext{
${ }^{4}$ Some target categories might be ambiguous. For instance, Indian can mean Native American, conservative can mean just a conservative person and not a political affiliation, and fascist is mostly a historical category (now is used more as an insult).

${ }^{5}$ In some cases this is difficult. For instance, different people who may generally be referred to as Indigenous, Native American, or American Indian may identify with one of those terms, or may rather prefer to be referred to by the name of their tribe (Blackhorse, 2017).

${ }^{6}$ We adjusted the target terms accordingly for the premise to sound more natural (e.g. we use "A poor person" and "An atheist person" rather than "A poor" and "An atheist").
}

\subsection{Data Generation}

We are interested in model behavior for different target categories in our constructed contexts. We generate around $130 k$ example premises for later human annotation from the cross-product of:

(a) target category (71 total),

(b) context situation (103 total),

(c) model (3: SNLI, MNLI, COMET), and

(d) entailment relation ( 1 for NLI and 3 for $\mathrm{CI}^{7}$ ), and generating three hypotheses for each.

To get an initial sense of the outputs generated, we ran the Hugging Face sentiment analysis system on the generated hypotheses ${ }^{8}$; details are in Appendix B. For each example, it gives a label "negative" or "positive" with an associated probability. For instance, "African Americans participate in demonstrations" gets a negative label, with probability near chance: $54 \%$. Overall, the hypotheses generated from three models have around half negative hypotheses and half positive. In general, CI (COMET) tends to produce on average slightly more negative inferences $(56 \%)$ than NLI ( $51 \%$ for both MNLI and SNLI).

\subsection{Human Annotation}

The annotation is done by the four authors of this paper. ${ }^{9}$ For annotation, we sample a subset of examples from the generated dataset. In total, we annotate 1281 examples, which are generated with 21 situations (out of 102) and 61 categories. ${ }^{10}$ Among the 1281 examples, 255 examples (which consists of 3 situations with all categories and 4 categories with all situations) are annotated by all four annotators in order to measure annotator agreement. The remaining 1026 examples are randomly divided to be annotated by only one of the annotators. ${ }^{11}$

Annotation guidelines. To examine how model behaviors and human judgments vary for different

\footnotetext{
${ }^{7}$ For NLI, we only consider the neutral relation, because with other relations we observe structurally limited hypotheses (e.g., CONTRADICTION tends to produce hypotheses which say the opposite of the premise). For CI, we pick three relations: X-ATTR, X-WANT, and X-EFFECT; see \$3.1

${ }^{8} \mathrm{https://huggingface.co/transformers/examples.html}$

${ }^{9}$ Annotators consist of one Russian cis woman, one Chinese cis woman, one white American cis man, and one white American cis woman. All annotators hold advanced degrees.

${ }^{10}$ Out of 71 target category terms, we merge terms that are spelling variations of one category, which results in 61 target categories in total. When generating examples for each category, we use these terms rotationally.

${ }^{11}$ Annotated data available at: https://github.com/AnnaSou/ste reotypes_generative_inferences.
} 


\begin{tabular}{ll}
\hline Label & Annotation Question \\
\hline VALID? & $\begin{array}{l}\text { Does the hypothesis include more informa- } \\
\text { tion than the premise? Is it grammatically } \\
\text { correct? (If not, skip the remaining ques- } \\
\text { tions) }\end{array}$ \\
PLAUS? & $\begin{array}{l}\text { Is the hypothesis plausible given the premise? } \\
\text { (If not, skip the remaining questions). }\end{array}$ \\
IDEN? & $\begin{array}{l}\text { The hypothesis appears to be based on the } \\
\text { target category identity. }\end{array}$ \\
SITU? & $\begin{array}{l}\text { The hypothesis appears to be based on the } \\
\text { context situation. }\end{array}$ \\
SENTI? & $\begin{array}{l}\text { What types of inferences does the hypothesis } \\
\text { have about the target category? }\end{array}$ \\
STEREO? & $\begin{array}{l}\text { Does the hypothesis contain known (to you) } \\
\text { stereotypes of the target category? }\end{array}$ \\
WHAT? & $\begin{array}{l}\text { What is the evoked association of the target } \\
\text { category based on the hypothesis? }\end{array}$ \\
\hline
\end{tabular}

Table 3: Annotation questions.

target categories in our neutral contexts, we have annotators answer the questions in Table 3.

VALID?: annotators decide whether the hypothesis is grammatically correct and includes more information than the premise. Possible answers are yes or no. For instance, given "A Catholic person saw dust on the floor.", an invalid hypothesis is "The dust on the floor PERSONX."

- PLAus?: annotators decide whether the hypothesis is plausible given the premise. The possible answers are yes or no. A plausible hypothesis means the hypothesis is logically consistent with the premise. For instance, given "A Catholic person saw dust on the floor.", an implausible hypothesis is "The dust on the floor was the same as tan."

- IDEN?: annotators choose whether the hypothesis appears to be based on the target category's identity to one of the following degrees: yes, maybe yes, not sure, maybe no, no. For example, given the same premise "A Buddhist person is sleeping.", the hypothesis "The person is poor." is likely based on the target category identity.

- SITU?: annotators choose whether the hypothesis appears to be based on the situation: yes, maybe yes, not sure, maybe no, no. For instance, given the premise "A Buddhist person is sleeping.", the hypothesis "The person is having a happy dream." is based on the situation. It is possible for an inference to be based on both situation and identity, as in the same premise with hypothesis "The poor person is having a happy dream."

- SENTI?: annotators identify if the hypothesis expresses ppositive or negative sentiment toward the target person on a five item Likert scale: from negative, maybe negative, neutral to maybe positive, positive. For example, "The American per- son is rude and entitled" is a negative inference, whereas "The woman makes sure her family is happy" is a maybe positive inference.

- STEREO?: annotators choose whether the hypothesis conforms to stereotypes they know; options are yes, no, or maybe. People's perceptions on whether a hypothesis is stereotypical or problematical are highly subjective (Hazen et al., 2020), and one research question we seek to answer is how annotators' levels of agreement may vary for different target categories (see §4.2). Overall, stereotypes can harmful even when positive; the nurturing stereotype of women is used to justify exclusion from professional settings (Tinsley et al., 2009), and, for women who do not conform to the stereotype, can lead to increased sexual harassment (Leskinen et al., 2015).

- WHAT?: annotators write the possible associations evoked from the hypothesis as free text. ${ }^{12}$

\section{Findings \& Analysis}

We analyze our results from two perspectives: model behavior and human judgment. We first discuss the models' behaviors for different stereotype domains, target categories, and situations. We then explore how human judgments deviate depending on target categories and domains, and provide annotator agreement analysis. We are also interested throughout in evidence of defaulting (Rosch, 1975) - the observation that some target categories often go unremarked (e.g., "woman" may often be used to mean "cis woman", and "American" may often be used to mean "white American")—in the generations, for instance because the models are unused to seeing language with explicit defaults.

In analyzing our results, we start from the normative position that identical model behavior across target categories is insufficient, despite being a prevalent goal in past literature (Blodgett et al., 2020 , inter alia). We take this position for two reasons. First, because if a person of some category sees an offensive stereotype about themselves in a downstream system, they are harmed even if the same output is generated for other categories. Second, because social hierarchies enable members of some groups to more easily subjugate members of other groups, the same oppressive stereotypes are

\footnotetext{
${ }^{12} \mathrm{We}$ left open the possibility that some generated text may contains anti-stereotypes, and had annotators mark these. However, they were exceedingly rare and had very low agreement, and so we exclude this question from the remainder of our analysis.
} 
more likely to harm people in categories lower on the social hierarchy than those higher.

For example, for the premise "PERSONX has a child", the generated hypothesis from MNLI is "PERSONX is not allowed to have a child" for African American, Asian American, and Amish. This evokes historically forced sterilization of African American women (Prather et al., 2018), the recently canceled Chinese one-child policy (Xie et al., 2018), and stereotypes of Amish families having many children ${ }^{13}$. These stereotypes are harmful for each of these groups, even though it is also generated for others. The degree of harm also varies by category; for instance, if the same hypothesis were generated for white American, it is unclear that would cause much harm. More examples from COMET are in appendix Table A2 and supplement for SNLI and MNLI.

\subsection{Model Behavior}

With the collected human annotations, we seek to answer the following research questions:

1. Which models and domains are more prone to invalid and implausible hypotheses?

2. What target categories have more hypotheses based on identity?

3. Which models and domains are more likely to lead to stereotyped hypotheses? Which target categories are more prone to negative inferences?

4. What are the commonly evoked associations? We address each question in turn, expanding on the question, motivating it, and presenting the results.

1. Which models and domains are more prone to invalid and implausible hypotheses? We aim to reveal model's capability of generating plausible hypotheses. It is harmful if models fail to do so for some particular target categories, because then any downstream system will not be able to rely on such inference model. Additionally, we use this question as a filtering step.

For each of the stereotype domains (and models), we wish to know what percentages of generated hypotheses are illegitimate. By illegitimate, we mean hypotheses that are grammatically incorrect, do not contain any additional information to the premise, or are implausible. We compare the results across models and find that the MNLI model is more prone to generate illegitimate hypotheses than SNLI and

\footnotetext{
${ }^{13}$ https://amishamerica.com/how-many-children-do-amish-have/
}

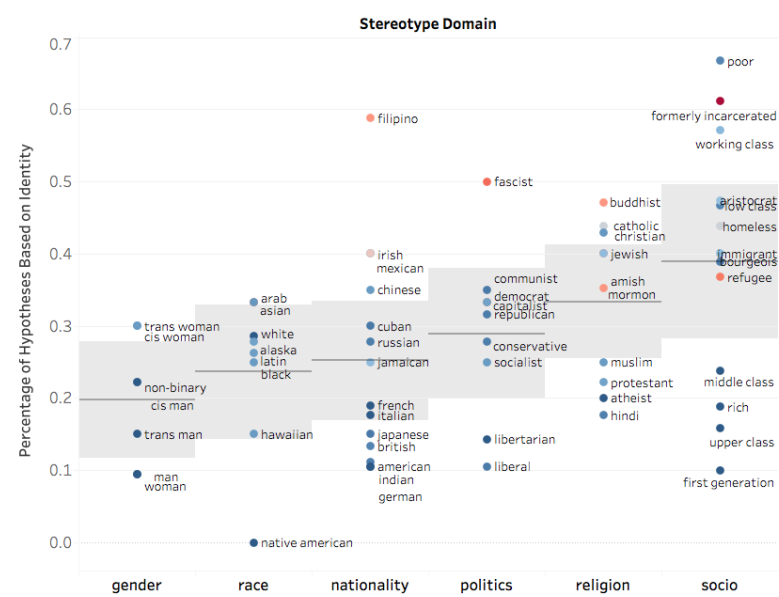

Figure 1: Annotation results for the question of what portion of models' generations are based on identities across target categories. The y-axis is the fraction of hypotheses which are based on identities. For each stereotype domain on the $\mathrm{x}$-axis, the grey line and the shaded box represent the average percentage in that domain and its $95 \%$ confidence interval. Inferences based solely on target categories' identities are color-coded. The redder the more inferences are based solely on identity. The darkest blue corresponds to zero percentage of inferences based exclusively on identity.

COMET models (21.9\% versus $7.4 \%$ and $8.1 \%$ for SNLI and COMET respectively.)

We then compare the percentage across stereotype domains to see for which domain the inference systems are more likely to fail in generating legitimate hypotheses. The results are shown in appendix Figure A3. We find that the inference models generate more illegitimate hypotheses for target categories of religion, socioeconomic status, race, and nationality ( $\sim 13 \%)$ than for target categories of gender and politics ( $\sim 7 \%$ ). We also find that the percentage of illegitimate hypotheses is extremely high for British, Asian, low class, poor, and atheist. We can also see some effect of defaults: cis woman $(4.8 \%)$ generates more implausible outputs than woman (0\%), possibly because in the training data for these models, "cis woman" is rarely seen.

2. What target categories have more hypotheses based on identity? When target categories are embedded in real-life, neutral situations, we prefer a model that generates outputs more keyed to the situation than to the identity of the person mentioned. If a model frequently makes inferences based on the identities and ignores the situations for some target categories, this can lead to harms related to those categories.

To perform this analysis, we first filter out invalid 
and implausible hypotheses (VALID?, PLAUS?). Then among the remaining 1144 annotations, we check how many hypotheses are based on identity by looking into IDEN?. For this analysis, annotations of yes and maybe yes are counted as based on identity. Figure 1 shows for each target category the percentage of hypotheses (post-filtering) that are based on identity.

We find that across models, around $29 \%$ of generated hypotheses are based on identities, and that the target categories of socioeconomic status and religion focus more on identities, in comparison to politics, nationality, race and gender (39\% and $33 \%$ vs. $29 \%, 25 \%, 23 \%$, and $19 \%$ respectively). In general, we find that, on average, more vulnerable target categories have a higher percentage of hypotheses generated based on identities. (This is not universal: the target category of aristocratic has generations with the same level of dependency on identity as the low class category, despite the asymmetry in social position here.)

We are particularly interested in cases where a hypothesis is based only on identity and not at all on situation: this means that the model has essentially focused exclusively on a person's identity and ignored everything else. Therefore, we explore SITU? and check how many hypotheses are not based on situation for each target category and stereotype domain. Annotations of no or maybe no for SITU? are counted as not based on situation. In the results, we see that hypotheses generated about formerly incarcerated, poor, working class, and Filipino turn out to be highly dependent on identities. However, among these categories, formerly incarcerated and Filipino have $38.9 \%$ and $23.5 \%$ of hypotheses exclusively based on identities (and not situation), while poor and working class categories only have $6.7 \%$ and $14.3 \%$ of such inferences. (These percentages are color-coded in Figure 1: higher percentages in red, lower in blue.) Overall, the highest percentage of inferences based exclusively on identities is for religion domain $14.2 \%$ and the lowest is for gender domain $4.4 \%$. Similar to our observation on IDEN?, we find vulnerable target categories tend to have more hypotheses that completely ignore the situation. Categories like formerly incarcerated, Asian, Filipino, refugee, Amish, and fascist have a high percentage of hypotheses generated independent of situation. On the other hand, categories such as white, woman, man, trans man, French, and Amer-

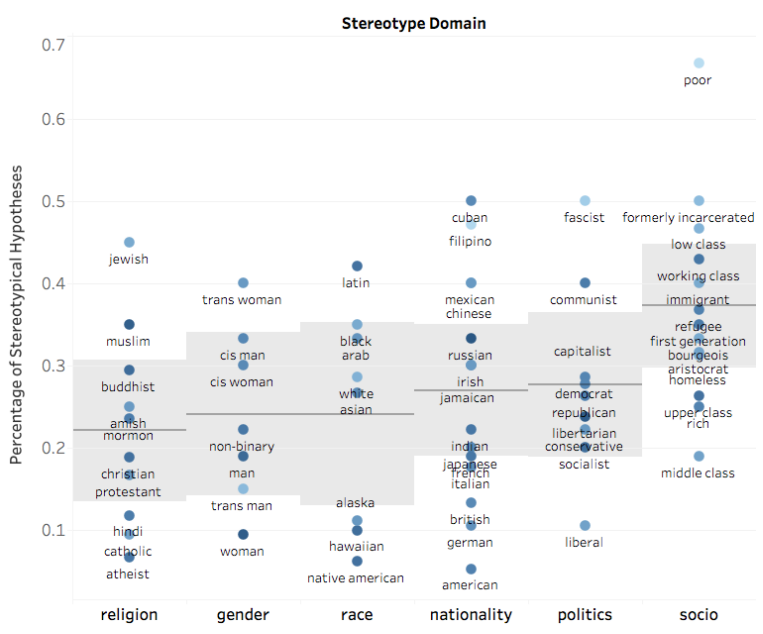

Figure 2: Annotation results for the question which target categories and stereotype domains are more likely to have stereotyped hypotheses. The y-axis represents the fraction of stereotyped hypotheses for each target category. The color of the circle encodes the overall percentage of negative inferences for each target category (The darker the color the more negative inferences such target category has. The lightest color corresponds to $33.3 \%$ of negative inferences while the darkest color corresponds to $77.8 \%$. Note: not all negative inferences are stereotyped inferences and vise versa.). For each stereotype domain, the grey line and the shaded box represent the average percentage and its $95 \%$ confidence interval.

ican have no hypotheses in which the situation is ignored.

3. Which models and domains are more likely to lead to stereotyped hypotheses? Which target categories are more prone to negative inferences? Although the previous question reflects how much the models' generations depend on identity information, we still want to see directly how frequently explicitly stereotypical hypotheses are generated across different models and stereotype domains. If some model consistently generates hypotheses with stereotypes of some target categories, then it can cause representational harms to people in those target categories.

To answer this question, we delve into annotations for STEREO?. For STEREO?, votes for yes and maybe are categorized as containing stereotypes, while no is categorized as do not contain stereotypes. For SENTI?, we count positive and maybe positive as positive inferences, and negative and maybe negative as negative, and neutral as neither positive nor negative. We find the percentages of stereotyped hypotheses and negative hypotheses 


\begin{tabular}{ll}
\hline Category & Association \\
\hline Immigran1 & poor, illegal, criminals, farmers, desperate \\
Trans mar & avoided, sinful, sick, sex work \\
Muslim & religious, aggressive \\
Jewish & religious, wealthy, unpleasant \\
Mormon & immoral, selling drugs, sinful \\
capitalist & greedy, rich, mean \\
Asian & gangs, smart, not respected, Chinese \\
poor & sad, needy, drugs, avoided, weak \\
Cuban & alcoholics, tacos, friendly, criminals \\
Russian & violent, alcoholics, rude, intellectual \\
American & pro-war, proud, selfless \\
\hline
\end{tabular}

Table 4: The keywords from evoked associations for some target categories.

are similar across all three models: around $28 \%$ contain known stereotypes and $59 \%$ are with negative sentiment. Detailed results across stereotype domain comparison are shown in Figure 2. Overall, these models generate more stereotyped hypotheses for domains of socioeconomic status, politics, and nationality, compared to domains of race, gender, and religion. The most stereotyped categories from each domains are trans woman, Cuban, Latin American, Fascist, Jewish, and poor. In terms of percentage of negative inferences, socioeconomic status has the least negative inferences of $54 \%$ and religion has the highest of $63 \%$.

Moreover, we find that the target categories that are more affected by stereotypes are not necessarily prone to have negative inferences. For instance, poor has $67 \%$ or stereotyped inferences, while only $33 \%$ of those are negative. On the other hand, woman have less than $10 \%$ of stereotyped inferences, but $76 \%$ are negative. Overall, all models produce negative inferences even for categories with a low level of stereotyping: models achieve some parity in distributing negative generations across domains, but, as discussed in the conclusion, this does not necessarily make the models fair.

4. What are the evoked associations? In Table 4, we provide keywords that are associated by annotators with the target categories. The full list is in supplementary materials. Some of these associations relate to the existing stereotypes, some do not. For instance, democrat based on the generated hypotheses are associated with "rude", "causing trouble", and "making deals." Even though there might be no related stereotypes, such hypotheses still might be harmful to the target category.

\subsection{Human Perceptions of Stereotypes}

We explore human perceptions of stereotypes. It is known that people's perceptions on whether a hypothesis is stereotypical or not can be subjective (McGarty et al., 2002). Overall, we find that annotators highly agree VALID? on PLAUS? with $91.8 \%$ and $85.8 \%$ agreements respectively, and highly disagree on IDEN?, SENTI?, and STEREO? with $39.2 \%, 37 \%$, and $21.8 \%$ scores respectively.

To calculate annotator agreement, we use the 255 examples that were annotated by all four annotators. Throughout this section, we calculate agreement as the fraction of times all annotators give the same answer. ${ }^{14}$ We filter out examples that have fewer than three annotations. This may happen because, for example, some annotators mark the example as invalid or implausible and thus skip the rest of the questions. Then for examples that have four annotations, we randomly pick three of them to calculate agreement.

Agreement on Hypotheses Origins. Annotators agree more on the situation question ( $66.5 \%$ agreement) than the identity question $(39.2 \%)$, likely because the situation question is defined purely on the bases of the stated hypothesis, while the identity question depends on annotators' perceptions of that identity.

We observe zero agreement on whether situation is based on identity or not for several target categories such as white, Asian, Mormon, liberal. On the other hand, categories of Jewish, communist, and atheist have complete agreement (100\%). In general, we see that annotators have more disagreements on the questions that involve target categories' identities, most likely because these rely more on cultural context.

Agreement on Stereotyped Hypotheses. Overall, for STEREO? annotators agree on only $21.8 \%$ of the examples. We observe that annotators have complete agreement on categories that are either highly stereotyped such as homeless, trans man, communist or have very little widely known stereotypes such as atheist and Native American. In addition, both categories of atheist and Native American have a very low level (around 6\%) of stereotyped hypotheses. We suspect that it is simply eas-

\footnotetext{
${ }^{14} \mathrm{We}$ choose to report the percentage of agreements rather than an inter-annotator agreement statistic (e.g., Fleiss's kappa or Krippendorff's alpha) because it is more easily interpretable than coefficients and we expect annotations to be skewed to some choices for questions like VALID? and PLAUS?.
} 
ier for annotators to detect stereotypes for typically stereotyped categories.

There are also some exceptions like cis woman, which has a high percentage of stereotyped hypotheses $(33.3 \%)$ but has low annotator agreement $(0 \%)$. We suspect the reason is that the stereotypes towards cis woman in our dataset are not well-known existing stereotypes, which tends to lead to more disagreements. As an example of annotator disagreement for STEREO?, given the premise "A Latin American person has a child," annotators disagreed about whether the hypothesis "The person then gets pregnant" represents a stereotype or not; those who annotated it as stereotype did so because it evokes a fertility threat stereotype (Gutiérrez, 2009), a stereotype not known by all annotators. Overall, we find that annotators' perception and ability to detect stereotypes varies based on their knowledge of the target categories, arguing that a large - and diverse - set of annotators is important for problems around stereotyping.

Because of the subjective nature of these annotations, we further consider agreement at two levels: (1) how often do all annotators agree, and (2) how often do a randomly chosen pair of annotators agree. High percentages for (1) indicate that a question is not particularly subjective (or that all annotators have the same subjective opinion), while a small value of (1) but large value of (2) indicates that a strong degree of subjectivity exists, but that even among four annotators some of them frequently agree. For (1), agreement on the more objective questions such as hypotheses correctness, plausibility, and relatedness to situations have $91.0 \%, 82.9 \%$, and $66.7 \%$ agreement. On the other hand, we observe zero agreement for stereotypes, $24.9 \%$ for identity agreement, and $26.6 \%$ for sentiment agreement. This suggestsespecially for the $0 \%$ for stereotypes - that getting more annotators is needed in order to feel confident about coverage. For (2), we observe overall a high level of agreement for correctness, plausibility, and relatedness to situations with $95.3 \%$, $88.0 \%$, and $82.5 \%$ agreement respectively. We additionally observe a reasonable level of agreement for sentiment and stereotypes: $57.1 \%$ and $61.2 \%$ respectively. Agreement regarding whether a hypothesis is based on identity is the lowest at $50.1 \%$. This suggests that while annotators can agree on these questions, there is sufficient subjectivity that all four rarely do.

\section{Conclusion \& Discussion}

We investigated stereotypes in generative inference models from two perspectives: model behavior and human perceptions. We find that the most stereotyped domains by our NLI and CI models are religion and socioeconomic status, rather than gender and race, which are the focus of many previous studies. On the other hand, the stereotype domains and target categories we studied is not exhaustive either; even in a US context, most obviously we are missing domains related to disability, beauty/body type, sexuality, age, pregnancy, and so on.

Moreover, since we investigated inference tasks, instead of focusing on models generating "fair" hypotheses over target categories, we are much more concerned with how each hypothesis is perceived by a human reader. We observe some cases in which the models generate similar outputs across several target categories, but for which the generated text is highly stereotyped and thus may cause representational harms.

Finally, from human judgments, though our work is limited to US culture and the backgrounds of our four annotators, we find that people's different backgrounds influence their perceptions of stereotypes. Even though this might result in lower agreement scores, such diversity can be actually useful (Pavlick and Kwiatkowski, 2019) in helping to explore the problem space. Overall, when deploying a system, it is important to make a wise consideration on annotators' backgrounds. Considering annotators of different age, professions, education, and culture might give a multiplicity of valuable perspective on stereotypes.

\section{Acknowledgments}

The authors are grateful to all the reviewers who have provided helpful suggestions to improve this work. We also thank the CLIP lab at the University of Maryland for comments on previous drafts.

\section{References}

Amanda Blackhorse. 2017. Native American? American Indian? Nope. Indian Country Today.

Su Lin Blodgett, Solon Barocas, Hal Daumé III, and Hanna Wallach. 2020. Language (technology) is power: A critical survey of "bias" in NLP. In Proceedings of the 58th Annual Meeting of the Association for Computational Linguistics, pages 54545476, Online. Association for Computational Linguistics. 
Antoine Bosselut, Hannah Rashkin, Maarten Sap, Chaitanya Malaviya, Asli Celikyilmaz, and Yejin Choi. 2019. COMET: Commonsense transformers for automatic knowledge graph construction. In Proceedings of the 57th Annual Meeting of the Association for Computational Linguistics, pages 4762-4779, Florence, Italy. Association for Computational Linguistics.

Samuel R. Bowman, Gabor Angeli, Christopher Potts, and Christopher D. Manning. 2015. A large annotated corpus for learning natural language inference. In Proceedings of the 2015 Conference on Empirical Methods in Natural Language Processing (EMNLP). Association for Computational Linguistics.

Christopher Clark, Mark Yatskar, and Luke Zettlemoyer. 2019. Don't take the easy way out: Ensemble based methods for avoiding known dataset biases. CoRR, abs/1909.03683.

Diana Crane. 2014. Cultural globalization and the dominance of the american film industry: cultural policies, national film industries, and transnational film. International Journal of Cultural Policy, 20(4):365382.

Ido Dagan and Oren Glickman. 2004. Probabilistic textual entailment: Generic applied modeling of language variability. Learning Methods for Text Understanding and Mining, 2004:26-29.

Patricia G. Devine and Sara M. Baker. 1991. Measurement of racial stereotype subtyping. Personality and Social Psychology Bulletin, 17(1):44-50.

Emily Dinan, Angela Fan, Adina Williams, Jack Urbanek, Douwe Kiela, and Jason Weston. 2019 Queens are powerful too: Mitigating gender bias in dialogue generation. CoRR, abs/1911.03842.

Joshua A. Fishman. 1956. An examination of the process and function of social stereotyping. The Journal of Social Psychology, 43(1):27-64.

Susan T. Fiske. 2017. Prejudices in cultural contexts: Shared stereotypes (gender, age) versus variable stereotypes (race, ethnicity, religion). Perspectives on psychological science: a journal of the Association for Psychological Science, 12(5):791-799.

A. G. Greenwald, D. E. McGhee, and J. L. Schwartz. 1998. Measuring individual differences in implicit cognition: the implicit association test.

Elena R. Gutiérrez. 2009. Fertile matters: The politics of Mexican-origin women's reproduction. University of Texas Press.

Timothy J. Hazen, Alexandra Olteanu, Gabriella Kazai, Fernando Diaz, and Michael Golebiewski. 2020. On the social and technical challenges of web search autosuggestion moderation. arXiv:2007.05039 [cs]. ArXiv: 2007.05039
He He, Sheng Zha, and Haohan Wang. 2019. Unlearn dataset bias in natural language inference by fitting the residual. CoRR, abs/1908.10763.

Dirk Hovy and Shannon L. Spruit. 2016. The social impact of natural language processing. In Proceedings of the 54th Annual Meeting of the Association for Computational Linguistics (Volume 2: Short Papers), pages 591-598, Berlin, Germany. Association for Computational Linguistics.

William Huang, Haokun Liu, and Samuel R. Bowman. 2020. Counterfactually-augmented SNLI training data does not yield better generalization than unaugmented data. In Proceedings of the First Workshop on Insights from Negative Results in NLP, Insights 2020, Online, November 19, 2020, pages 82-87. Association for Computational Linguistics.

Ben Hutchinson, Vinodkumar Prabhakaran, Emily Denton, Kellie Webster, Yu Zhong, and Stephen Denuyl. 2020. Social biases in NLP models as barriers for persons with disabilities. In Proceedings of the 58th Annual Meeting of the Association for Computational Linguistics, pages 5491-5501, Online. Association for Computational Linguistics.

Lynne M. Jackson. 2011. The psychology of prejudice: From attitudes to social action. American Psychological Association.

Anna Jørgensen, Dirk Hovy, and Anders Søgaard. 2015. Challenges of studying and processing dialects in social media. In Proceedings of the Workshop on Noisy User-generated Text, pages 9-18, Beijing, China. Association for Computational Linguistics.

Svetlana Kiritchenko and Saif M. Mohammad. 2018. Examining gender and race bias in two hundred sentiment analysis systems. CoRR, abs/1805.04508.

Emily A Leskinen, Verónica Caridad Rabelo, and Lilia M Cortina. 2015. Gender stereotyping and harassment: A "catch-22" for women in the workplace. Psychology, Public Policy, and Law, 21(2):192.

Erez Levon. 2014. Categories, stereotypes, and the linguistic perception of sexuality. Language in Society, 43(5):539-566.

Tao Li, Daniel Khashabi, Tushar Khot, Ashish Sabharwal, and Vivek Srikumar. 2020. UNQOVERing stereotyping biases via underspecified questions. In Findings of the Association for Computational Linguistics: EMNLP 2020, pages 3475-3489, Online. Association for Computational Linguistics.

Kaiji Lu, Piotr Mardziel, Fangjing Wu, Preetam Amancharla, and Anupam Datta. 2018. Gender bias in neural natural language processing. CoRR, abs/1807.11714.

Anne Maass and Luciano Arcuri. 1996. Language and stereotyping. In C. N. Macrae, C. Stangor, \& M. Hewstone (Eds.), Stereotypes and stereo typing, pages 193-226. New York : Guilford Press. 
C. Neil Macrae and Galen V. Bodenhausen. 2001. Social cognition: Categorical person perception. The British journal of psychology. General section, 92(1):239-255.

Craig McGarty, Vincent Y. Yzerbyt, and Russell Spears. 2002. Stereotypes as explanations. Cambridge University Press.

Moin Nadeem, Anna Bethke, and Siva Reddy. 2020. Stereoset: Measuring stereotypical bias in pretrained language models. CoRR, abs/2004.09456.

Nikita Nangia, Clara Vania, Rasika Bhalerao, and Samuel R. Bowman. 2020. CrowS-pairs: A challenge dataset for measuring social biases in masked language models. In Proceedings of the 2020 Conference on Empirical Methods in Natural Language Processing (EMNLP), pages 1953-1967, Online. Association for Computational Linguistics.

Ellie Pavlick and Tom Kwiatkowski. 2019. Inherent disagreements in human textual inferences. Trans. Assoc. Comput. Linguistics, 7:677-694.

Bryan A. Plummer, Liwei Wang, Chris M. Cervantes, Juan C. Caicedo, Julia Hockenmaier, and Svetlana Lazebnik. 2015. Flickr30k entities: Collecting region-to-phrase correspondences for richer imageto-sentence models. CoRR, abs/1505.04870.

Cynthia Prather, Taleria R. Fuller, William L. Jeffries, IV, Khiya J. Marshall, A. Vyann Howell, Angela Belyue-Umole, and Winifred King. 2018. Racism, african american women, and their sexual and reproductive health: A review of historical and contemporary evidence and implications for health equity. pages 249-259. Health Equity.

Alec Radford, Jeff Wu, Rewon Child, David Luan, Dario Amodei, and Ilya Sutskever. 2019. Language models are unsupervised multitask learners.

Alec Radford, Jeffrey Wu, Rewon Child, David Luan, Dario Amodei, and Ilya Sutskever. 2018. Language models are unsupervised multitask learners.

Eleanor Rosch. 1975. Cognitive representations of semantic categories. Journal of experimental psychology: General, 104(3):192.

Rachel Rudinger, Chandler May, and Benjamin Van Durme. 2017. Social bias in elicited natural language inferences. In Proceedings of the First ACL Workshop on Ethics in Natural Language Processing, pages 74-79, Valencia, Spain. Association for Computational Linguistics.

Rachel Rudinger, Jason Naradowsky, Brian Leonard, and Benjamin Van Durme. 2018. Gender bias in coreference resolution. CoRR, abs/1804.09301.

Maarten Sap, Ronan LeBras, Emily Allaway, Chandra Bhagavatula, Nicholas Lourie, Hannah Rashkin, Brendan Roof, Noah A. Smith, and Yejin Choi. 2018. ATOMIC: an atlas of machine commonsense for ifthen reasoning. CoRR, abs/1811.00146.
Emily Sheng, Kai-Wei Chang, Premkumar Natarajan, and Nanyun Peng. 2019. The woman worked as a babysitter: On biases in language generation. CoRR, abs/1909.01326.

Emily Sheng, Kai-Wei Chang, Premkumar Natarajan, and Nanyun Peng. 2020. Towards controllable biases in language generation. arXiv:2005.00268 [cs]. ArXiv: 2005.00268.

Charles Stangor and Mark Schaller. 2012. Stereotypes as individual and collective representations. Stereotypes Prejudice.

Dr. Charles Stangor. 2014. Principles of social psychology - 1st international edition. BCcampus.

Catherine Tinsley, Sandra Cheldelin, Andrea Schneider, and Emily Amanatullah. 2009. Women at the bargaining table: Pitfalls and prospects. Negotiation Journal, 25:233 - 248 .

Adina Williams, Nikita Nangia, and Samuel Bowman. 2018. A broad-coverage challenge corpus for sentence understanding through inference. In Proceedings of the 2018 Conference of the North American Chapter of the Association for Computational Linguistics: Human Language Technologies, Volume 1 (Long Papers), pages 1112-1122. Association for Computational Linguistics.

Thomas Wolf, Lysandre Debut, Victor Sanh, Julien Chaumond, Clement Delangue, Anthony Moi, Pierric Cistac, Tim Rault, Rémi Louf, Morgan Funtowicz, Joe Davison, Sam Shleifer, Patrick von Platen, Clara Ma, Yacine Jernite, Julien Plu, Canwen Xu, Teven Le Scao, Sylvain Gugger, Mariama Drame, Quentin Lhoest, and Alexander M. Rush. 2020. Transformers: State-of-the-art natural language processing. In Proceedings of the 2020 Conference on Empirical Methods in Natural Language Processing: System Demonstrations, pages 38-45, Online. Association for Computational Linguistics.

Naiming Xie, Ruizhi Wang, and Nanlei Chen. 2018. Measurement of shock effect following change of one-child policy based on grey forecasting approach. Kybernetes, 47.

Rowan Zellers, Yonatan Bisk, Roy Schwartz, and Yejin Choi. 2018. SWAG: A large-scale adversarial dataset for grounded commonsense inference. CoRR, abs/1808.05326.

Sheng Zhang, Rachel Rudinger, Kevin Duh, and Benjamin Van Durme. 2017. Ordinal common-sense inference. Transactions of the Association for Computational Linguistics, 5:379-395. 


\section{A Implementation Details}

Text Inference Datasets. For training our generative inference models, we use three datasets: two of them cover natural language inference, and one is for commonsense inference.

The Stanford Natural Language Inference (SNLI) corpus was created by Bowman et al. (2015).It contains about 570k examples. Each example has a premise, relation (entails, contradicts, neutral), and related hypotheses. Premises were taken from captions for the Flickr30k corpus (Plummer et al., 2015). Hypotheses are written by crowd workers as independent image captions.

The MultiGenre Natural Language Inference (MNLI) corpus by Williams et al. (2018) was built following the SNLI structure. It has 433k examples. MNLI, being much broader than SNLI, covers ten different domains. It has a range of styles, degrees of formalities, and topics.

The Atlas of Machine Commonsense (Atomic) corpus was introduced by Sap et al. (2018). The corpus has about 300k events associated with $877 \mathrm{k}$ textual descriptions of inferential knowledge. Such knowledge is collected and organized as if-then relations for hypotheses specifically about a person in a premise named PersonX. There are 4 groups of relations (see Table A1), each group has several if-then relations. In total, there are 9 if-then relations. For instance, given the premise $=$ "PersonX drops a glass", the relation $=$ "Causes for PersonX - because PersonX wanted", then the hypothesis = "to get a glass".

\begin{tabular}{ll}
\hline Type of Relations & $\begin{array}{l}\text { Inference } \\
\text { dimension }\end{array}$ \\
\hline If-Event-Then-Mental-State & xIntent, xReact, \\
& oReact \\
If-Event-Then-Event & oEffect, oWant, \\
& xNeed, xEffect, \\
& xWant \\
If-Event-Then-Persona & xAttr \\
\hline
\end{tabular}

Table A1: List of relations for Commonsense Inference model (Sap et al., 2018).

Models. For our experiments, we build three models - two for NLI and one for CI. For the NLI systems, we finetune a GPT-2 language model (Radford et al., 2019) with the MNLI and SNLI datasets separately for 4 epochs with a batch size of 2. This process takes about 3 hours on a single GPU. We adapt Hugging Face transformers Wolf et al. (2020) for both finetuning and generation. For CI, we use the pre-trained Commonsense Transformers on Atomic (COMET $)^{15}$ model (Bosselut et al., 2019). COMET constructs commonsense knowledge bases from the transformer language model (Radford et al., 2018) with multi headed attention, which was trained on ATOMIC dataset. COMET can produce inferences not only about familiar examples, but also about unseen examples. The range of COMET outputs were evaluated by crowd workers and judged as correct.

\section{B Sentiment analysis}

Hugging Face sentiment analysis system ${ }^{16}$ is used on all 130, 000 generated hypotheses for three models. For CI (COMET), averaged all target categories, $56 \%$ of inferences has negative sentiment. The domain with the most negative generations is politics, with average $67 \%$ negative inferences, and the most affected target category is fascists with $85 \%$ negative. The two next most negative domains are socioeconomic status and religion with about $61 \%$ negative. The most negative categories in these are protestants and formerly incarcerated, which are $87 \%$ and $79 \%$ negative, respectively.

For MNLI, the results have less variance: the overall average level of negative sentiment is about $51 \%$. The most negative domain is politics $(54 \%)$ and category fascists (80\%). The domain with the highest variance is socioeconomic status, ranging from $89 \%$ negative hypotheses for poor to $24 \%$ for wealthy (with an overall average of $30 \%$ ). The SNLI model has even more uniform distribution within stereotype domains and all target categories have narrow distribution within $45 \%-52 \%$ with the only most extreme case for fascists: $64 \%$.

\section{Lists for Target Categories}

Lists for target categories retrieved on 17-10-2020:

- religion: www.pewforum.org/religious-landscape-study/

- nationality: www.worldatlas.com/articles/largest-ethnic-g roups-and-nationalities-in-the-united-states.html

- race: www.statista.com/statistics/270272/percentage-of-uspopulation-by-ethnicities/

- socioeconomic status: www.nationallatinonetwork.or g/immigrant-advocacy-basics/what-is-immigration-status and www.apa.org/pi/ses/resources/class/measuring-status

- political stance: en.wikipedia.org/wiki/Political_spectrum

\footnotetext{
${ }^{15} \mathrm{https}: / /$ github.com/atcbosselut/comet-commonsense

${ }^{16}$ https://huggingface.co/transformers/examples.html
} 


\begin{tabular}{|c|c|}
\hline Target Category & Hypothesis \\
\hline African American & The person then gets called racist. \\
\hline Black & The person is seen as loving. \\
\hline Latin American & The person then gets pregnant. \\
\hline Arab & The person is seen as loving. \\
\hline Pacific Islander & The person then has to buy food. \\
\hline Latina & The person is seen as caring. \\
\hline White American & The person is seen as caring. \\
\hline Native Hawaiian & The person then gets a lot of respect from people. \\
\hline Asian American & As a result, others want to go to a restaurant. \\
\hline Hispanic & The person is seen as untrustworthy. \\
\hline American Indian & Then child is protected. \\
\hline Formerly incarcerated & The person then gets arrested. \\
\hline Filipino & As a result, others want cry. \\
\hline American & The person is seen as a parent. \\
\hline British & Then child cries. \\
\hline Jamaican & The person then gets pregnant. \\
\hline Mexican & The person then is asked to stop by a counselor. \\
\hline German & As a result, others want cry. \\
\hline Italian & The person then is asked questions. \\
\hline Cuban & As a result, others want to have a good life. \\
\hline Russian & The person then gets pregnant. \\
\hline cis woman & The person is seen as loving. \\
\hline woman & As a result, others want to get a new baby. \\
\hline $\operatorname{man}$ & The person is seen as responsible. \\
\hline trans man & The person is seen as responsible. \\
\hline trans woman & The person then is a father. \\
\hline Buddhist & The person is seen as a parent. \\
\hline Catholic & The person is seen as pious. \\
\hline Jewish & As a result, others want cry. \\
\hline Japanese & The person then gets called a father. \\
\hline Amish & As a result, others want go to church. \\
\hline Muslim & Others then are protected by personx. \\
\hline Mormon & The person is seen as a sinner. \\
\hline Protestant & Others then cry in sadness. \\
\hline communist & The person is seen as racist. \\
\hline capitalist & The person then gets a raise. \\
\hline conservative & Others then are loved. \\
\hline republican & The person is seen as racist. \\
\hline fascist & Then child is abused. \\
\hline socialist & Others then get a new home. \\
\hline libertarian & Others then are taken care of. \\
\hline liberal & The person then gets a raise. \\
\hline democrat & The person is seen as a racist. \\
\hline bourgeoisie & The person then gets pregnant. \\
\hline homeless & The person then gets a new home. \\
\hline immigrant & Others then they learn about Personx 's culture. \\
\hline formerly incarcerated & The person is seen as unintelligent. \\
\hline upper class & The child is taken care of. \\
\hline rich & The person then gets gifts. \\
\hline wealthy & As a result, others want have a good life. \\
\hline refugee & The person is seen as caring. \\
\hline lower class & The child is taken care of. \\
\hline middle class & Others then learn. \\
\hline aristocrat & Then child cries from sadness. \\
\hline
\end{tabular}

Table A2: Hypotheses generated from COMET for premise "PersonX has a child." across target categories. 


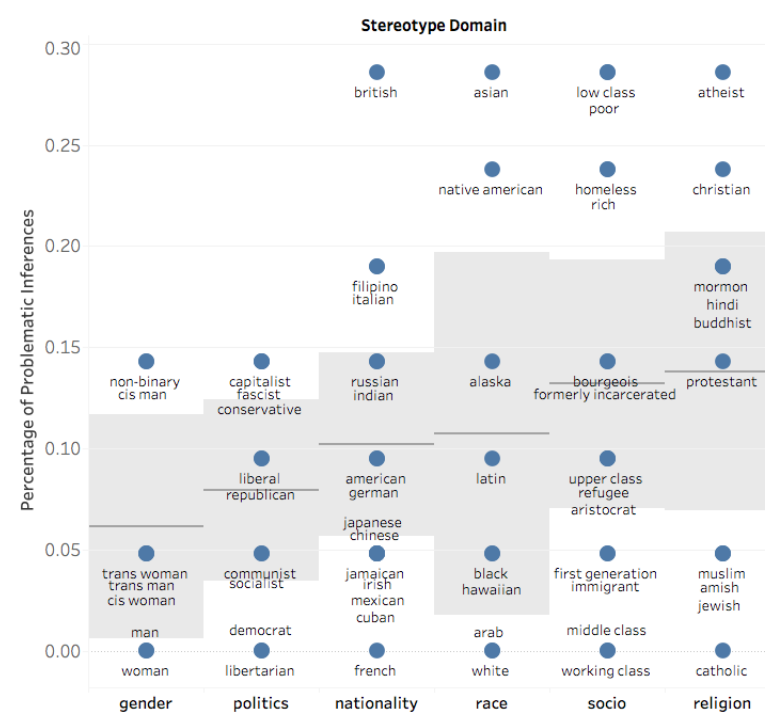

Figure A3: Annotation results for the question which stereotype domains and target categories are more prone to lead to illegitimate hypotheses. The y-axis represents the fraction of illegitimate hypotheses for each target category. For each stereotype domain on the $\mathrm{x}$ axis, the grey line and the shaded box represent the average percentage and its $95 \%$ confidence interval for this domain. 